\title{
Clinico-Epidemiological Study of Bacterial Meningitis in Pediatrics Age Group of 0-5 Years in Odisha, India
}

\author{
C. P. Khuntia ${ }^{1}$, S. K. Kar ${ }^{1}$, B. Dwibedi ${ }^{2}$ and Snehasish Mishra ${ }^{3}$ \\ ${ }^{1}$ Regional Medical Research Center (ICMR), Bhubaneswar, Odisha, India \\ ${ }^{2}$ All India Institute of Medical Science, Bhubaneswar, Odisha, India \\ ${ }^{3}$ School of Biotechnology, KIIT Deemed University, Bhubaneswar, Odisha, India \\ *Corresponding author
}

\section{Keywords}

Bacterial meningitis, pediatric, Haemophilus influenzae type b, Streptococcus pneumoniae

Article Info

\section{Accepted:}

12 May 2021

Available Online:

10 June 2021

\section{A B S T R A C T}

Meningitis is a very serious and fatal infection of the meanings associated with high mortality and morbidity in children. There is absolutely very little data available on this serious infectious disease in eastern region of India (Odisha). Viewing the present scenario the study is taken up to determine the incidence and etiological profile of bacterial meningitis with reference to the complications among the pediatric age group of one month to five years. The present study was conducted in a pediatric referral hospital of Odisha for a period of 18 months. Suspected meningitis cases were enrolled considering the clinical manifestation of bacterial meningitis. Out of total admitted cases 634 cases were suspected with meningitis while 61 cases were confirmed bacterial meningitis. Most (59.6\%) cases belonged to less than 1year age group and males outnumbered the females by a ratio of 2:1. Streptococcus pneumoniae was most common pathogen cases (57.6\%) followed by Haemophilus influenzae type b (26.2\%).

\section{Introduction}

Bacterial meningitis, an infection of the membranes (meninges) and cerebrospinal fluid (CSF) surrounding the brain and spinal cord, is a major cause of death and disability worldwide (1). Bacterial meningitis is associated with high mortality and morbidity worldwide, with an estimated 16 million cases in 2013, causing 1.6 million years lived with disability each year (2). According to Indian records 0.5 to $2.6 \%$ of hospital admissions are of Acute Bacterial Meningitis. In developed countries (with best available facilities), the cases fatality rate is $10 \%$ while it is $16-30 \%$ in India (3). Three bacteria account for most cases in infants and young children i.e Haemophilus influenzae type b (Hib), Streptococcus pneumoniae (Pneumococcus), and Neisseria meningitidis (Meningococcus). Although safe and effective vaccines have existed for years, vaccination rates remain 
suboptimal in South Asia and the impact of vaccine implementation has been substantial among low- and middle-income countries like India. Therefore these pathogens are still the major cause for bacterial meningitis hence forth early diagnosis, clinical signs and symptoms are of utmost important for detecting and monitoring the complications of meningitis.

\section{Materials and Methods}

This was a cross sectional study undertaken in a pediatric tertiary care hospital and teaching institution in Cuttack, Odisha, India. Ethical approval was taken from the Institutional Ethics Committee.

Inclusion criteria for suspected meningitis was all the admitted cases during the study period below 5 years of age with the history of sudden onset of fever more than $38.5^{\circ} \mathrm{C}$ rectal or more than $38.0^{\circ} \mathrm{C}$ axillary and the presence of one or more of the following such as neck stiffness, altered consciousness, meningeal sign. The purpose of the study was explained to the parent or guardian of the child and informed consent was taken before enrolling them in the study. Detailed clinical examination was done. Blood samples and cerebrospinal fluid samples were taken from the patients and analysed.

\section{Results and Discussion}

A total no of 634 children (1-59 months) of suspected meningitis cases were admitted to the hospital during this study period and 61 cases were diagnosed as meningitis.

Out of the total confirmed cases 14 samples were positive by latex agglutination where as six samples were positive for Hib, seven for $S$. pneumoniae and one for group B Streptococcus. Bacterial cultures were confirmed in 13 cases. The isolates are
S.pneumoniae, Hib, Pseudomonas aeruginosa, Klebseilla pneumoniae, E.coli, Salmonella typhi and Staphylococcus aureus. In Multiplex Real Time PCR 49 cases were confirmed out of which 35 S. pneumoniae and 14 cases were positive for Hib (Fig 6). The incidence of $S$. pneumoniae was highest among the common agents of bacterial meningitis (Fig 4). Culture and latex positive samples of Streptoccus pneumoniae and Hib were also found positive in RT PCR.

Out of these 61 laboratory confirmed cases of bacterial meningitis during the study period, $56(74.7 \%)$ were males and $19(24.3 \%)$ were females (Fig 1). The highest incidence of bacterial meningitis was present in infants less than one year of age which accounts to about 40 cases out of $61(53.3 \%)$. The incidence is more than half of the total confirmed cases followed by children less than 2 years of age which constituted 15 cases (20\%) (Fig 3). When the data is analyzed by paired sample $\mathrm{T}$ test showed confirmed case of meningitis is positively correlated with the sex and the difference is statistically significant $(\mathrm{p}<0.001)$.

The most marked seasonal variation was observed with a high incidence in the winter season (November to February) followed by summer (March to June). When the cases were split according to the time of presentation the highest incidence was observed in the month of December (Fig 2).

The most common outcome of the treatment was complete recovery which was observed in $45(74 \%$ ) out of 61 cases (Fig 5). During the study period 10 deaths were recorded due to bacterial meningitis, thus giving a case fatality rate (CFR) of $13.3 \%$. These fatal cases were then further analyzed by breakup into the various age groups. Most of the fatal cases were in infants or young adults. When age specific CFRs were studied, infants had the highest CFR of $41.6 \%$. 


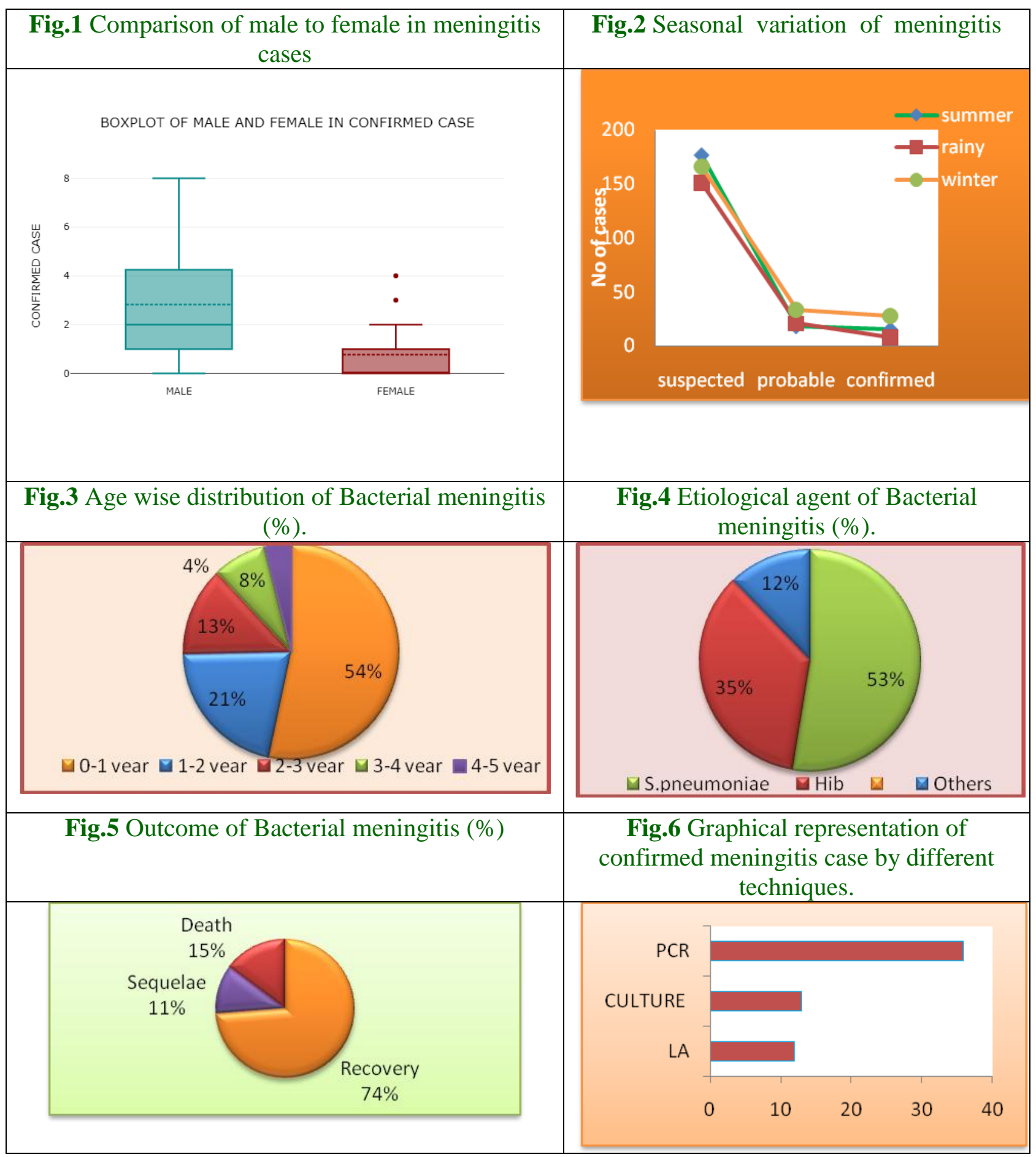

This was followed by the children between the age groups of 1 to 2 years. A higher CFR of $30 \%$ was observed in these age groups. A gender-wise breakdown of the fatal cases showed that more deaths were recorded in males then females. Results show that 8 out of
56 male patients died due to bacterial meningitis during the study period 2 deaths in a total of 49 females that presented during the same period. The study is a hospital based study and conducted in a tertiary health care centre. So the true prevalence and incidence of 
bacterial meningitis are difficult to define in populace due to the fact that most of the cases treated with broad spectrum antibiotics before reaching the hospital yielding in less no of culture isolates. The study showed a marked male predominance (male to female ratio of 1.7:1) in both suspected and confirmed cases of meningitis. Throughout the various age groups the trend of male predominance also tends to persist. Various large scale studies conducted worldwide show a higher incidence in males. Several studies conducted in India also revealed the male predominance which corroborated with our present investigation (4). Lower health seeking and utilization rates among females in India can also explain lower proportion of female patients in our study (5). Bacterial meningitis is generally a disease of childhood. In this study the most commonly affected age group was found to be the infants Data showed that almost half of the cases $(53.3 \%)$ were less than I year age group. But as per hospital based study in India in children aged 1 month to 5 years suffering from acute bacterial meningitis, $77.7 \%$ were below the age of 1 year (3). The higher rate of meningitis in early age children may be because of the underdeveloped immune system. During the study period 10 deaths were recorded due to bacterial meningitis, thus giving a case fatality rate (CFR) of $13.3 \%$ and when age specific CFRs were studied, infants had the highest CFR of $41.6 \%$. Different study conducted in India and other developed countries reported the case fatality rate as 16 to $30 \%$ (3). The cases of bacterial meningitis mainly occurred in winter and spring seasons $(6,7)$ which is corroborated with the present scenario where the most marked seasonal variation was observed with a high incidence in the winter followed by summer.

LAT test detected bacterial antigens in $23 \%$ cases of bacterial meningitis, with the most common organism being $\mathrm{S}$. pneumoniae followed by Hib. This finding was similar to that in other studies, where LAT was positive in $15.4 \%$ cases of bacterial meningitis (8). Isolation rate of Streptococcus pneumoniae and Hib were less in the study. This may be because of fastidious nature of the organisms, vaccine implementation against these organisms or antibiotic treatment prior to lumber puncture. The CSF becomes sterilized within 4 hours of parenteral antibiotic treatment in case of pneumococcal meningitis (9). Real time PCR analysis found more sensitive than Latex test and culture where the positivity rate is $59 \%$. Latex detects bacterial capsular antigens for the identification of the isolates 10 while culture identification is dependent on the recovery of a viable isolate from a specimen (10). In contrast, rt-PCR targets specific genes and does not require a viable pathogen (11). The rt-PCR method offers several other advantages, including the provision of results within hours, high throughput, and high sensitivity and specificity (11).

The incidence of meningitis is still high probably due to low practice of vaccination against common causative agents such as $S$. pneumoniae, $H$. Influenzae type $\mathrm{b}$ in India. Diagnosis of bacterial meningitis can be established by CSF Latex agglutination test, biochemistry, cytology. Now a day RT PCR plays a vital role in detection of meningitis irrespective of prior antibiotic administration and low bacterial load but its high cost is a major constraint for developing countries like India. Therefore prompt diagnosis and aggressive management practices are utmost priorities to reduce the morbidity and mortality of pediatric age group along with in prevention of complications.

\section{Acknowledgement}

The authors are thankful to the concerned Pediatricians of the hospital, Parent and the patient and DG, ICMR for cooperation. 


\section{References}

1. World Health Organization (WHO), 2011 Meningitis manual. : Laboratory Methods for the Diagnosis of Meningitis caused by Neisseria meningitidis, Streptococcus pneumoniae, and Haemophilus influenzae, second edition.

2. Global Burden of Disease Study 2013 Collaborators (2015). Global, regional, and national incidence, prevalence, and years lived with disability for 301 acute and chronic diseases and injuries in 188 countries, 1990-2013: a systematic analysis for the Global Burden of Disease Study 2013. The Lancet; 386(9995): 743800

3. Chinchankar N, Mane M, Bhave S, Bapat S, Bavdekar A, Pandit A, et al., Diagnosis and outcome of acute bacterial meningitis in early childhood. Indian Pediatr 2002;39:914-21.

4. Bhat B V, Verma I C, Puri R K, Srinivasan S, Nalini P. A profile of pyogenic meningitis in children. J Indian Med Assoc., 1991; 89: 224-7.

5. Malhotra C, Do Y K. Socio-economic disparities in health system responsiveness in India. Health policy and planning, 2012; 28(2): 197-205.

6. Schlech WF 3rd, Ward JI, Band JD, Hightower A, Fraser DW, Broome CV. Bacterial meningitis in the United States,
1978 through 1981. The National Bacterial meningitis surveillance study. JAMA 1985;253:1749-54.

7. Farag H F, Fatt ah Addel M M, Youssri A M. Epidemiological, clinical and prognostic profi le of acute bacterial meningitis among children in Alexandria, Egypt. Indian $\mathbf{J}$ Med Microbiol 2005;23:95-101.

8. Surinder K, Bineeta K, Megha M. Latex particle agglutination testas an adjunct to the diagnosis of bacterial meningitis. Indian J Med Microbiol 2007;25:395-7.

9. Keith A V. Cartwright. Bacterial Meningitis. Topley and Wilson's microbiology and microbial infections. Vol. 10. Italy: Hodder Arnold; 2005.p. 555-7.

10. Jones C H, Mohamed N, Rojas E, et al., Comparison of phenotypic and genotypic approaches to capsule typing of Neisseria meningitidis by use of invasive and carriage isolate collections. J Clin Microbiol 2016; 54:25-34.

11. Vuong J, Collard J M, Whaley M J, et al., Development of real-time PCR methods for the detection of bacterial meningitis pathogens without DNA extraction. PLoS One 2016; 11:e0147765. https://apps.who.int/iris/ handle/10665/70765. Accessed 25 July 2019.

\section{How to cite this article:}

Khuntia, C. P., S. K. Kar, B. Dwibedi and Snehasish Mishra. 2021. Clinico-Epidemiological Study of Bacterial Meningitis in Pediatrics Age Group of 0-5 Years in Odisha, India. Int.J.Curr.Microbiol.App.Sci. 10(06): 283-287. doi: https://doi.org/10.20546/ijcmas.2021.1006.029 